\title{
Inhibition of tumourigenicity of small cell lung cancer cells by suppressing Id3 expression
}

\author{
LALEH KAMALIAN, SHIVA S. FOROOTAN, ZHENG Z. BAO, YU ZHANG, \\ JOHN R. GOSNEY, CHRISTOPHER S. FOSTER and YOUQIANG KE \\ Molecular Pathology Laboratory, School of Cancer Studies, University of Liverpool,
6th Floor, Duncan Building, Daulby Street, Liverpool, L69 3GA, UK
}

Received April 9, 2010; Accepted May 20, 2010

DOI: 10.3892/ijo_00000708

\begin{abstract}
Id3 is over-expressed in small cell lung cancer (SCLC). To test whether the tumourigenicity of SCLC cells can be inhibited by suppressing Id 3 expression, we transfected siRNA into SCLC cell line GLC-19 and established two sublines (G-Id3-1 and G-Id3-7) which expressed only 30\% of the level of Id3 measured in control transfectants. Suppression of Id3 expression in both G-Id3-1 and G-Id3-7 cells produced significant reductions in proliferation rates and in numbers of colonies formed in soft agar assay. When G-Id3-1, G-Id3-7 and the control transfectants were inoculated subcutaneously into 3 groups (8 each) of nude mice, respectively, all (100\%) inoculated animals produced tumours. Although there was no difference in tumour incidents amongst the 3 groups, significant reductions were observed in both size and weight of tumours produced by either G-Id3-1 or G-Id3-7 cells. While the final average volume of tumours produced in control group was $1012.1 \pm 394 \mathrm{~mm}^{3}$, it was significantly reduced $(\mathrm{p}<0.001, \mathrm{p}<0.01)$ by $2.1-$ and 2.9 -fold to $475.7 \pm 167 \mathrm{~mm}^{3}$ and $354.3 \pm 218 \mathrm{~mm}^{3}$ in groups inoculated with G-Id3-1 and G-Id3-7 cells, respectively. Similar differences were also observed in average weight of tumours. Upon induction of apoptosis by cytotoxin camptothecin, the percentages of apoptotic cells in G-Id3-1 and G-Id3-7 were, respectively $>2.4$-fold higher than that in control. The results in this study suggest that highly expressed Id3 in SCLC cells may be an important therapeutic target for tumour suppression.
\end{abstract}

\section{Introduction}

Small cell lung cancer (SCLC) is one of the most aggressive human cancers, accounting for $\sim 16 \%$ of all pulmonary tumours

Correspondence to: Professor Y. Ke, Molecular Pathology Laboratory, School of Cancer Studies, University of Liverpool, 6th Floor, Duncan Building, Daulby Street, Liverpool L69 3GA, UK

E-mail: yqk@liverpool.ac.uk

Key words: small cell lung cancer, Id3 expression in England and Wales in 2006 (England National Office for Statistics and Welsh Cancer Intelligence and Surveillance Unit). Despite all efforts in understanding the molecular biology of this tumour, the underlying genetic alteration responsible for its unique disseminative behaviour is not clear. Previously, we showed that inhibitors of differentiation/ DNA binding (Id) family proteins were generally overexpressed in malignant SCLC cell lines and tissue samples when compared to their normal bronchial epithelial counterparts and suggested that they might be involved in carcinogenesis and progression of SCLC (1). Amongst the Id family proteins overexpressed in SCLC, Id3 exhibited the most pronounced change in its expression level.

Id3 is a member of Id family proteins, a group of transcription factors belonging to the basic helix-loop-helix (b-HLH) family. To date, four members of the Id family have been identified and accordingly named as Id1, 2, 3, and 4 (2). In order to activate transcription, bHLH proteins bind to each other forming homodimers or heterodimers. This enables them to bind to special sequences (CANNTG) called E-boxes in the promoter region of corresponding genes (3). Unlike the other classes, class V bHLH or Id proteins lack the DNA binding domain. Instead, they act as negative dominant regulators of other bHLH factors namely class I and II and some members of class III $(4,5)$. By binding to these factors, Id proteins inhibit them from making dimers and binding to E-box of DNA to inhibit transcription (3). Class I and II factors regulate the transcription of cell cycle genes $(6,7)$ and class II factors regulate cell-type specific genes (8). In this way Id proteins inhibit cell cycle progression and differentiation. Id proteins can also target some of class III HLH factors as well as some transcription factors not containing HLH domain such as ETS (9), PAX (10) and pRB $(11,12)$. When Id 2 is in excess, it can inhibit $\mathrm{RB}$ protein directly and result in cell cycle progression. Inversely, Id1 and Id3 mainly target ETS factors through which they inhibit CDK inhibitors such as $\mathrm{p} 21^{\mathrm{cip} 1}, \mathrm{p} 27^{\mathrm{kip} 1}$ and p16 $6^{\mathrm{INK} 4 \mathrm{~A}}$. These inhibitors sequestrate CDKs and therefore stop them from phosphorylating $\mathrm{pRB}$, hence having an indirect anti-proliferation effect (13). Id3 specifically regulates P27 through an ETS domain factor called ELK1 (14). Id1 and Id3 have also been identified as downstream targets of MYC (15). MYC binds to E box in Id3 promoter region and increases its transcription (16). 
Being involved in such important regulatory pathways makes Id proteins likely candidates to play a part in tumourigenesis of cancers. Id proteins were reported to be dysregulated in carcinomas of many organs, including prostate (17), colon (18), liver (19), breast (20), cervix (21), pancreas (22), ovary (23), thyroid (24), and the squamous carcinoma of the head and neck (25). Also, Id2 and Id3 were over-expressed in $\mathrm{T}$ cell leukemia (26). More recently, the increased expression in SCLC and its possible prognostic significance were investigated and the increased Id2 in cytoplasm was found to be significantly related to increased survival of patients (1).

Previously, there has been strong evidence indicating possible roles for Id3 protein in the pathogenesis of other cancers such as hepatocellular carcinoma, prostate cancer and colorectal adenocarcinoma. In head and neck squamous cell carcinoma, forced expression of Id 3 reduced invasiveness of tumour cells via interfering with transcription of matrix metalloproteinase 2 (MMP2) (27). Inversely, in pancreatic carcinoma where, Id1, Id 2 and Id3 were over-expressed, Id1/Id3 double knockdown reduced metastatic potential of malignant cell lines (28). However, it is not yet clear whether the increased Id3 in SCLC can be used as a target for tumour suppression.

In this study, the short-silencing RNA (si-RNA) technique was used to reduce Id 3 expression in the highly malignant SCLC cell line GLC-19 so as to establish two cell lines with $70 \%$ reduction in Id 3 protein level compared to the control and the parental cell line. GLC-19 cells were shown to express the highest level of the Id3 protein amongst the 10 SCLC cell lines tested previously (1). The Id3-suppressed cells derived from GLC-19 were subjected to a series of bioassays in vitro and in vivo to test the effect of reducing Id3 expression on the malignant characteristics of the cells. In addition, the possible molecular mechanism involved in the biological function of Id 3 was investigated by relating its reduced expression to apoptosis induced by camptothecin.

\section{Materials and methods}

Cell lines and culture condition. SCLC GLC-19 cell line was used for gene knockdown and normal bronchial epithelial cell line Beas-2b was used as a control. Both cell lines were obtained from the cell line archive of the Pathology Department of Liverpool University. GLC-19 and its siRNA transfected cell lines were grown as suspension culture while Beas-2b as an adherent culture. All cultured cells were maintained in RPMI-1640 (PAA Cell Culture Co.) supplemented with 10\% (v/v) FCS (BioSera, UK), L-glutamine ( $2 \mathrm{mmol} / \mathrm{l})$, penicillin $(100 \mathrm{U} / \mathrm{ml})$ and streptomycin $(100 \mu \mathrm{g} / \mathrm{ml})$. For stably transfected cell lines during and after the selection process, geneticin G418 was added to the culture medium with a concentration of $1 \mathrm{mg} / \mathrm{ml}$ to select the cells harbouring the transfected DNA constructs.

Construction of siRNA expressing vector. Three unique $21 \mathrm{nt}$ sequences (all have $<70 \%$ GC content) located at different sites of coding region of Id 3 cDNA were designed as possible siRNA sites, using the method described on the Biotech Company Ambion, (Cambridgeshire, UK) website (www. ambion.com). The uniqueness of the sequences was confirmed by BLAST search to ensure there was no significant homology towards other human or mouse DNA sequences. Each DNA fragment consisted of two 21-bp sequences in sense and antisense orientations separated by a 9-bp loop sequence (TTCAAGAGA) in the middle and capped at the 3'-end with a poly $\mathrm{T}$ termination sequence flanked by a HindIII restriction recognition site. At the 5'-end, the short DNA molecule was flanked by a poly-A termination sequence preceded by a BamH1 restriction recognition site. The short DNA was commercially synthesised (Invitrogen Life Technologies, Strathclyde, UK) and purchased as two separate oligonucleotides. These two complementary oligonucleotides (positive and negative strands) were annealed in sense and antisense directions to form a double stranded DNA fragment which was then cloned into the pSilencer 4.1 CMV neo plasmid (Ambion) by HindIII and BamH1 sites, transformed and amplified in E. coli (DH5- $\alpha$ ) cells. Three selected siRNA sequences from different regions of Id 3 cDNA are shown in Fig. 1. A scrambled RNA molecule was also cloned into the same plasmid to form a control expression construct. Successful insertions of short DNA into the plasmid and the orientation of the short DNA were confirmed, respectively, by automatic sequencing using an internal pSilencer 4.1 generic sequence primer (5'-ATTAACCCTCAGTAAAGGGA-3').

Transient transfection. Transfection of the siRNA-expressing plasmids into the highly malignant GLC-19 cells was performed with the Lipofectamine 2000 transfection reagent (Invitrogen, CA, USA) following the manufacturer's instructions. The DNA constructs containing different siRNA inserts were first dissolved in Lipofectamine at a DNA/Lipofectamine ratio of 1/2.5 (w/v), diluted in Opti-MEM I Reduced Serum and then added to $2 \mathrm{ml}$ of cell suspension samples at density of $1 \times 10^{6}$ cells $/ \mathrm{ml}$. Transfection reaction took place in a tissue culture incubator at $37^{\circ} \mathrm{C}, 5 \% \mathrm{CO}_{2}$ and $95 \%$ humidity. The transfection reaction was stopped and cells lysed for total RNA extraction at 24,48 and $72 \mathrm{~h}$, respectively.

Total RNA extraction and cDNA synthesis. Total RNA was extracted and purified using RNeasy Mini extraction kit (Qiagen, CA, USA). RT-PCR was performed with the SuperScript ${ }^{\mathrm{TM}}$ III First-Strand System (Invitrogen) following the manufacturer's instruction. The first strand of the cDNA was synthesized using $0.5 \mu \mathrm{g}$ of total RNA as start material and an oligo-dT primer $(50 \mu \mathrm{M})$ at $65^{\circ} \mathrm{C}$. The second DNA strand was synthesised based on the first strand at $55^{\circ} \mathrm{C}$ for $1 \mathrm{~h}$. The synthesised cDNA was used as template for further analysis with quantitative PCR or Q-PCR.

$Q-P C R$. The quantitative PCR was performed using the above described synthetic cDNA as template and reaction reagents with a Brilliant SYBR Green QPCR mastermix kit were purchased commercially (Stratagene, USA). The sequence of the forward Id3 primer was 5'-CGCGTCATCGACTACAT TCT-3' and that of the reverse primer was 5'-GAGCTC GGCTGTCTGGAT-3'. Amplification of B-actin gene was performed in parallel to normalize possible loading errors. The cycle threshold $(\mathrm{Ct})$ was used to calculate relative amounts of Id3 mRNA. The $\Delta \Delta \mathrm{Ct}$ method was employed for relative 


\begin{tabular}{|c|c|c|c|c|c|}
\hline & GATCTGGGGT GCTGCCAGGA & AAAAGCAAAT & TCTGGAAGTT & AATGGTTTTG & AGTGA \\
\hline 61 & AAATCCTTGC TGGCGGAGAG & GCCCGCCTCT & CCCCGGTATC & AGCGCTTCCT & CATTCTTTGA \\
\hline 1 & ATCCGCGGCT CCGCGGTCTT & CGGCGTCAGA & CCAGCCGGAG & GAAGCCTGTT & TGCAATTTAA \\
\hline 1 & GCGGGCTGTG AACGCCCAGG & GCCGGCGGGG & GCGGGGCCGA & - GGCGGGCCAT & TTTGAATAAA \\
\hline & GAGGCGTGCC TTCCAGGCAG & GCTCTATAAG & TGACCGCCGC & GGCGAGCGTG & CGCGCGTTGC \\
\hline 1 & AGGTCACTGT AGCGGACTTC & TTTTGGTTTT & СтTTСTстTт & GGGGCACCTC & TGGACTCACT \\
\hline & CCCAGCATG AAGGCGCTGA & GCCCGGTGCG & CGGCTGCTAC & GAGGCGGTGT & GCTGCCTGTC \\
\hline 1 & $\begin{array}{cc}\overrightarrow{4} & \text { Sequence-1 } \\
\text { GGAACGCAGT } & \text { CTGGCCATCG }\end{array}$ & $\stackrel{ }{\mathrm{CCCGGGGCCG}}$ & AGGGAAGGGC & CCGGCAGCTG & AGGAGCCGCT \\
\hline 31 & GCTTGCTG GACGACATGA $\vec{A}$ & $\begin{array}{r}\text { Sequen } \\
\text { ACCACTGCTA }\end{array}$ & $\stackrel{c e-2}{\text { CTCCCGCCTG }}$ & CGGGAACTGG & TACCCGGAGT \\
\hline 11 & $\underline{\mathrm{CCCC}}$ & GCCAGGTGG $\vec{A}$ & $\begin{array}{r}\text { Squen } \\
\text { AATCCTACAG }\end{array}$ & CGCGTCATCG & ACTA \\
\hline 1 & CGACCTGCAG GTAGTCCTGG & CCGAGCCAGC & CCCTGGACCC & CCTGATGGCC & СССАССТTCC \\
\hline $\begin{array}{l}61 \\
21\end{array}$ & $\begin{array}{l}\text { CATCCAGACA GCCGAGCTCG } \\
\text { CCACTGACTC GGCCGTGTCC }\end{array}$ & $\begin{array}{l}\text { CTCCGGAACT } \\
\text { TGACACCTCC }\end{array}$ & $\begin{array}{l}\text { TGTCATCTCC } \\
\text { AGAACGCAGG }\end{array}$ & $\begin{array}{l}\text { AACGACAAAA } \\
\text { TGCTGGCGCC }\end{array}$ & GGAGCTTTTG \\
\hline 1 & GGGACCCCGG GAACCTCTCC & TGCCGGAAGC & CGGACGGCAG & GGATGGGCCC & CAACTTCGCC \\
\hline 41 & CTGCCCACTT GACTTCACCA & ААТСССТTСC & TGGAGACTAA & АCCTGGTGCT & CAGGAGCGAA \\
\hline 1 & GGACTGTGAA CTTGTAGCCT & GAAGAGCCAG & AGCTAGCTCT & GGCCACCAGC & TGGGCGACGT \\
\hline 61 & CACCCTGCTC CCACCCCACC & CCCAAGTTCT & AAGGTCTTTT & CAGAGCGTGG & AGGTGTGGAA \\
\hline 21 & GGAGTGGCTG CTCTCCAAAC & TATGCCAAGG & CGGCGGCAGA & GCTGGTCTTC & TGGTCTCCTT \\
\hline 081 & GGAGAAAGGT TCTGTTGCCC & TGATTTATGA & ACTCTATAAT & AGAGTATATA & GGTTTTGTAC \\
\hline 41 & СTTTTTTACA GGAAGGTGAC & TTTCTGTAAC & AATGCGATGT & АТАTTAAACT & TTTTATAAAA \\
\hline & & & & & \\
\hline
\end{tabular}

Figure 1. Selection of nucleotide sequences for silencing Id 3 gene. Underlined area is the CDS region of Id3 cDNA. Three high-lighted areas are selected regions for silencing Id 3 expression and are named sequences 1, 2 and 3, respectively.

quantification of gene expression. The Ct values of Id3 gene from triplicate PCR reactions were normalized to the levels of $\beta$-actin from the same c-DNA preparations. The average $\mathrm{Ct}$ for each gene was calculated by subtracting the $\mathrm{Ct}$ of the sample RNA from that of the control RNA (from control transfectants). The fold change in expression was calculated using the equation: $2 \Delta \mathrm{Ct}^{\text {cont }}-\Delta \mathrm{Ct}^{\text {sample }}$.

Stable transfection of siRNA Id 3 silencing construct. The construct exhibited the most effective suppression on Id3 expression in transient transfection experiments was stably transfected into GLC-19 cells using the same procedure and the same reagents as those used for transient transfection. The construct containing scrambled RNA was also transfected to generate a pool of control transfectants. After 24-h incubation of the reaction mixture, the cells in transfection reactions were transferred into a selection medium (normal medium containing $1 \mathrm{mg} / \mathrm{ml} \mathrm{G} 418$ ) for selections of the successful transfectants. When the colonies of transfectants were visible, the control cells were mixed together to form a pool of control transfectants. In order to obtain single colonies, the si-Id3 transfected cells growing in suspension were sub-cultured into a nitrocellulose semisolid ClonaCell-TSC (StemCell Technologies, UK) medium containing $1 \mathrm{mg} / \mathrm{ml} \mathrm{G} 418$. Ten single colonies were separated from the semisolid medium and cultured again in the selective medium (normal medium containing $1 \mathrm{mg} / \mathrm{ml} \mathrm{G} 418$ ) to form 10 separate cell lines.

Detection of protein expression in cultured cells. Expression of Id1, Id2 and Id 3 proteins in transfected cell lines was detected by Western blotting using ECL light-emitting non-radioactive kit (Amersham Pharmacia Biotech., UK) as described previously (29). Total protein in each sample $(50 \mu \mathrm{g})$ was quantified with a Coomassie Protein Assay Reagent kit (Pierce, IL, USA). Protein extracts prepared from different cell lines were subjected to SDS-PAGE in 15\% (w/v) polyacrylamide gels. The membrane was first incubated with preblocking reagents and then incubated with anti-human Id1, Id2 or Id3 protein polyclonal antibodies (Santa Cruz Biotechnology, Santa Cruz, USA) respectively at $1 / 100$ dilution overnight at $4^{\circ} \mathrm{C}$. The membrane was then incubated with horseradish peroxidase-conjugated swine anti-rabbit antibody (Dako, UK), the bound antibodies were revealed by chemiluminescence (ECL, Amersham) and recorded on Kodak XAR-5 film. Intensities of the protein bands were determined by scanning densitometry using an Alpha-Imager 2000 software (Alpha Innotech, Cannock, UK). The values of the bands were obtained by measuring the areas below the peaks. Relative levels of Id protein expression in different transfected cell lines were determined by comparing the intensities of 
the bands of the control cells. Blots were incubated with a specific anti-human $B$-actin monoclonal antibody (Sigma, UK) to correct the possible loading errors.

Proliferation assay. Effect of suppressed Id3 expression on cellular proliferation was measured by a CellTiter $96 \mathrm{AQ}_{\text {ueous }}$ One Solution Reagent (Promega, USA) which contains a tetrazolium compound [3-(4,5-dimethylthiazol-2-yl)-5(3carboxymethoxyphenyl)-2-(4-sulfophenyl)-2H-tetrazolium, inner salt; MTS] and an electron coupling reagent (phenazine ethosulfate; PES). The si-Id3 transfectant lines (G-Id3-1 and G-Id3-7) and the control transfectants were cultured in normal RPMI-1640 medium supplemented by $1 \mathrm{mg} / \mathrm{ml} \mathrm{G} 418$ in 6-well plates in triplicates. Each single well contained 5,000 cells in $1 \mathrm{ml}$ medium. In every 48 -h of 8 -day experimental period $100 \mu \mathrm{l}$ of sample in each well were transferred into wells of another 96-well micro-titer plate. Cells were disaggregated by pipetting and $20 \mu \mathrm{l}$ of MTS solution was added to each well. The plate was incubated at $37^{\circ} \mathrm{C}$ for $4 \mathrm{~h}$ to form a soluble coloured formazan product. The optical absorbance of each sample was measured with a Multiskan MS (Lab Systems Multiskan MS, Finland) plate reader using a 490-nm filter. The values of optical absorbance were compared with the standard curves established with serially-diluted cells. The number of cells represented by optical absorbance was converted by regression analysis.

Soft agar assay for tumourigenicity. Soft agar assay was performed to test the ability of si-Id3 transfected cells to produce colonies in an anchorage-independent environment as an indication of their tumourigenicity $(30,31)$. To start the assay, the bottom of each well in a 24-well plate was cast (at $4^{\circ} \mathrm{C}$ ) with $0.5 \mathrm{ml}$ of basement gel layer consisted of equal amounts of $2 \%$ low melting agar (LMA, Bioproducts, UK) and RPMI-1640 medium to make a gel of $1 \%$ agar. The cultured cells were harvested and suspended in LMA-RPMI containing $0.7 \%$ agar at a density of 10,000 cells $/ \mathrm{ml}$. From this mixture, $0.5 \mathrm{ml}$ (containing 5,000 cells) was poured onto the top of the preset basement gel layer and incubated at $4^{\circ} \mathrm{C}$ for solidification for $15 \mathrm{~min}$. Once set, the cells in the plates were incubated in a culture oven at $37^{\circ} \mathrm{C}$ under $5 \% \mathrm{CO}_{2}$ and $95 \%$ humidity for 2 weeks. The colonies developed in each well were counted and the tumourigenicity of each cell line was decided by the number of colonies formed in the soft agarose gel in the 2-week experimental period.

Nude mouse assay. To test the tumourigenicity in vivo, the transfectant cells were inoculated in Bulb/c male nude mice to produce tumours. For each animal, $5 \times 10^{6}$ cells in $200 \mu 1$ of an equal amount of matrigel/cell suspension mixture were injected subcutaneously in the shoulder area. Mice were weighed two times a week to monitor the body weight and the sites of injection were checked for signs of tumour growth. When tumours started to appear, three dimensions (length, width and depth) of tumours were measured using a calliper. On the day 20 of injection, animals were sacrificed using a $\mathrm{CO}_{2}$ chamber. From each animal, the tumour was dissected, measured and weighted. Tumours were assessed every 2/3 days and the volumes were calculated by a formula of: Volume $=$ $\pi / 6(\mathrm{H} \mathrm{x} \mathrm{W})^{3 / 2} 0.67(32,33)$ where $\mathrm{H}$ and $\mathrm{W}$ stands for height and width in millimetres respectively. All animal experiments were conducted under UKCCCR guidelines with Home Office Project Licence PPL40/2270 to Professor Y. Ke.

Measurement of the proportion of cells undergoing apoptosis. The percentage of cells undergoing apoptosis (programmed cell death) was measured using an Annexin V-FITC apoptosis detection kit (BioVision, UK), following the manufacturer's instructions. Degree of apoptosis was measured by analysing Annexin V-FITC binding detected by a FITC detector (FL1) and PI staining detected by a phycoerythrin emission signal detector (FL2). After the re-suspension of cell pellets containing $4 \times 10^{6}$ cells in binding buffer, $5 \mu \mathrm{l}$ of Annexin V-FITC and $5 \mu \mathrm{l}$ of propidium iodide were added to the cell/buffer mixture and incubated at room temperature for $10 \mathrm{~min}$ in dark and then run through an Epics XL MCL flow cytometer (Beckman Coulter, USA) to detect apoptotic cells. Using control cell line, adjustments were made in Scatter gram, FL1 and FL3 histograms to exclude cell debris and large particles. The viable and apoptotic cells were represented by B and C (horizontal lines) regions, respectively, in FL1 log histogram.

\section{Results}

Transient transfection of siRNA. To find out which of the 3 selected si-RNA sequences can produce the most effective suppression on Id 3 expression, all 3 molecules were transiently transfected into the highly malignant SCLC cell line GLC-19 and the effect of these sequences on the level of Id3 mRNA expression was measured using Q-PCR. Relative Id3 mRNA levels in control cells and in cells transiently transfected with 3 different siRNAs for 24,48 , and $72 \mathrm{~h}$, respectively are shown in Fig. 2A. When the level of Id3 mRNA in the untreated parental GLC-19 cells was set at 1, levels in other 3 groups of cells transfected with 3 siRNA sequences respectively were reduced by $20-80 \%$. Sequence- 2 and -3 produced almost the same level of Id 3 mRNA reduction at all 3 time-points and both of them exhibited a better suppression effect than sequence-1. Thus either sequence- 2 or -3 can be selected as an effective suppressor and hence sequence- 3 was picked up and used as an inhibitor for $\operatorname{Id} 3$ expression.

Stable transfection. The construct containing sequence-3 was transfected into GLC-19 cells, 10 colonies were isolated from the transfectants and each of them was cultured separately to form an individual cell line. These new transfectant lines were named G-Id3-1, 2, $3 \ldots$ to -10 , respectively. Relative Id3 mRNA levels in parental cell line GLC-19, control transfectants, the normal bronchi epithelial cell line Bease-2b and the 10 different new transfectant lines are shown in Fig. 2B. When the level of Id3 mRNA in parental GLC-19 cells was set at 1 , similar level was measured in the control cells. The level (1.05) of Id3 mRNA in G-Id3-2 was similar to that in GLC-19 cells, indicating no suppression was achieved. While the levels of Id3 mRNA in G-Id3-1 and G-Id3-5 cells were reduced to 0.35 and 0.55 respectively, those in other 7 new cell lines (ranged from 0.11 to 0.16 ) were remarkably reduced by 7-9-fold to a similar or even slightly lower level (0.14) measured in the normal bronchi epithelial cells Bease-2B. 

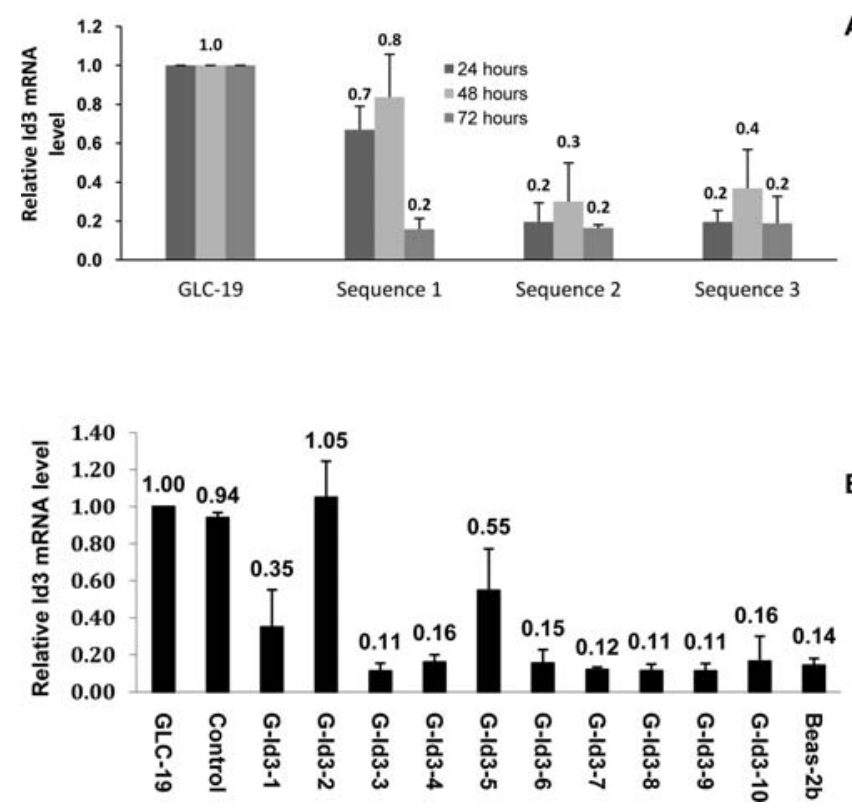

Figure 2. The effect of transient or stable transfection of siRNA on levels of Id3 mRNA expression in GLC-19 cells. (A) Relative Id3 mRNA levels in control cells and in cells transiently transfected with 3 different siRNAs for 24,48 , and $72 \mathrm{~h}$, respectively. The level of Id 3 mRNA in control cells was set at 1. Levels of Id3 mRNA in cells transfected transiently with different siRNAs at different time points were calculated by relating to that in the control. (B) Relative Id3 mRNA levels in parental GLC-19 cells, control transfectants, and in 10 different sublines established from 10 different single colonies isolated respectively from the stably-transfected GLC-19 cells with sequence 3. The level of Id3 mRNA in parental GLC-19 cells was set at 1 , levels in other cells were calculated by relating to that in parental cells. The level of Id 3 mRNA in benign Bease-2b cells was also measured as an additional control.

The effect of stable siRNA transfection on expression of Id3 and other Id-proteins in GLC-19 cells, control transfectants, and 3 representative new transfectant lines was analysed by Western blotting and the results are shown in Fig. 3A. Further quantitative assessment on the relative levels of Id3 protein expression is shown in Fig. 3B. When the level of Id3 protein in parental GLC-19 cells was set at 1, a similar level (0.97) was measured in the control transfectants. For the 3 new transfectant lines, the highest Id3 protein level (1.08) was measured in G-Id3-3 cells. Very similar Id3 protein levels were expressed in G-Id3-1 (0.32) and G-Id3-7 (0.33).

The possible effect of suppressing Id 3 expression on the expression of other Id-family proteins Id1 and Id 2 was investigated by detecting their expression with Western blot and the results are shown in Fig. 3C. When the levels of Id1 and Id 2 proteins in the control cells are set at 1 , their relative levels were 0.98 and 0.92 in G-Id3-1 cells, respectively, and 0.91 and 1.02 in G-Id3-7 cells, respectively. Thus, the levels of Id 1 and Id 2 expressed in the transfectant cell line G-Id3-1 and G-Id3-7 were very similar to that in the control.

Inhibition of Id3 expression reduced cell proliferation. The effect of reducing Id3 expression on proliferation rates of different cells was assessed by a proliferation assay and the results are shown in Fig. 4A. Starting with the same number $(5,000)$ of cells for each tested cell line, the number of cells on day 8 of the experiment was $47407 \pm 2035$ and $45510 \pm 2704$ in parental cell line and in the control transfectants,
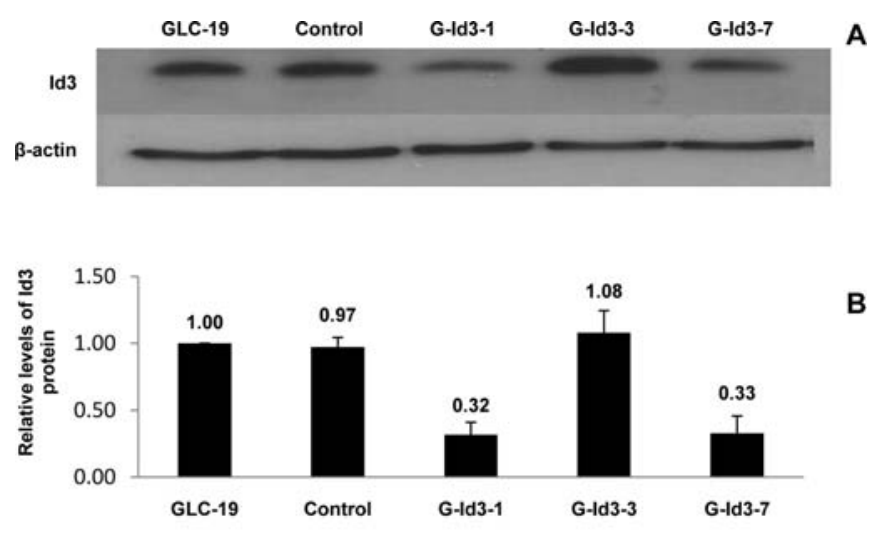

B

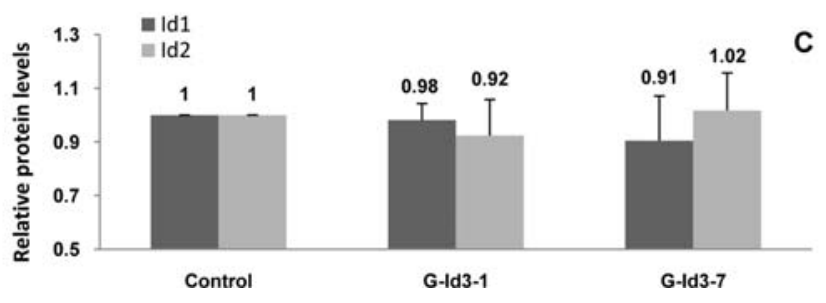

Figure 3. The effect of stable siRNA transfection on expression of Id3 and other Id-proteins. (A) Western blot analysis of Id3 protein expression in GLC-19 cells, control transfectants and 3 sublines established from sequence-3-transfectant clones. (B) Relative levels of Id3 protein expressed in GLC-19 cells, control transfectants, G-Id3-1 and G-Id3-7 cells. The level of Id3 protein in parental GLC-19 cells was set at 1 and levels in other cells were calculated by relating to that in parental cells. Results are obtained from 3 separate measurements (mean \pm SD). (C) Relative levels of Id1 and Id 2 protein expression in control transfectants, G-Id3-1 and G-Id3-7 cells. Protein levels of Id1 and Id2 in control cells are set at 1 and those in other cells are, respectively, calculated by relating to levels in control cells. Results are obtained from 3 separate measurements (mean \pm SD).

respectively; whereas in both G-Id3-1 and G-Id3-7 lines, the cell number was $19042 \pm 1946$ and $16437 \pm 978$, respectively, a $>2$-fold reduction. Further analyses showed that the reductions in cell numbers between the control and either GId3-1 or G-Id3-7 cells were statistically significant (Student's t-test, $\mathrm{p}<0.001, \mathrm{p}<0.001)$, whereas the difference in cell numbers was significant neither between G-Id3-1 and G-Id3-7 cells, nor between the parental GLC-19 and the control cells.

The anchorage-independent growth of different cells in soft agar. The ability of si-Id3 transfected cells to produce colonies in an anchorage-independent environment as an indication for tumourigenicity was tested in low melting temperature soft agar assay and a representative plate from each test cell line is shown in Fig. 4B. The total numbers of colonies produced by different cells are shown in Fig. 4C. After growing in soft agar for 2 weeks, the control transfectants produced an average of $94 \pm 13$ colonies in 3 separate tests, similar to that $(91 \pm 13)$ produced by the parental GLC- 19 cells. In comparing with the control cells, numbers of colonies produced by G-Id3-1 and G-Id3-7 cells were significantly (Student's t-test, $\mathrm{p}<0.001$ and $\mathrm{p}<0.01$ ) reduced by 3.3 -fold to $28 \pm 6$ and by 1.7 -fold to $53 \pm 7$, respectively.

Tumourigenicity in nude mouse. The control transfectants, G-Id3-1 and G-Id3-7 cells were inoculated, respectively, into 


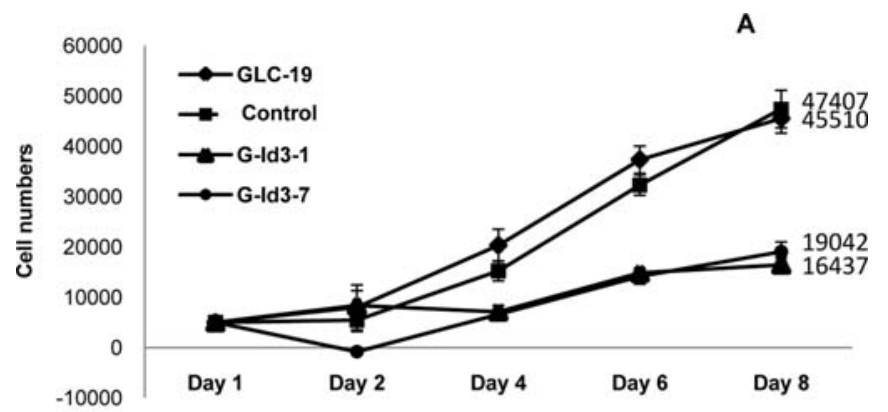

B
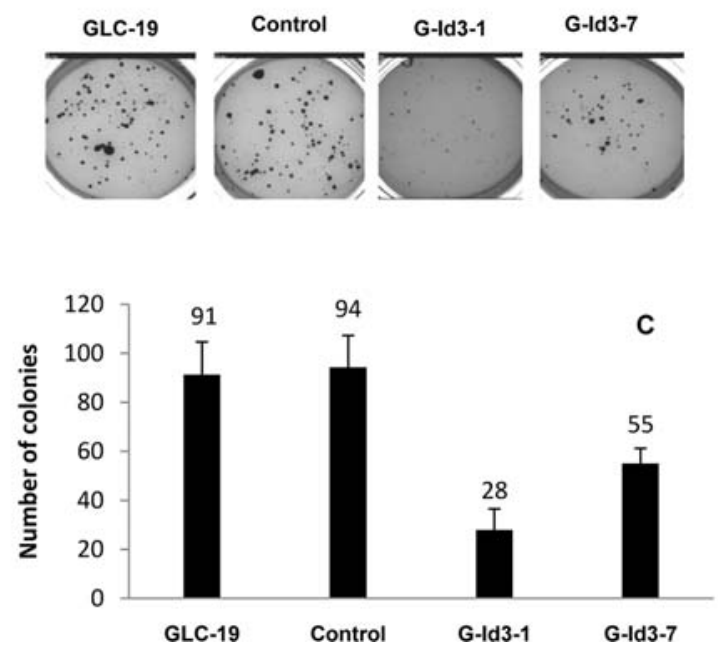

Figure 4. Proliferation rate and anchorage-independent growth of different cell lines in vitro. (A) Proliferation rates of parental GLC-19 cells, control transfectants, G-Id3-1 and G-Id3-7 cells. Experiments started with 5,000 cells for each cell line. Results are obtained from triplicate samples (mean \pm SD). (B) Anchorage-independent growth of different cell lines in soft agar. (C) Number of colonies formed by different cell lines in soft agar assay. Assays started with 5,000 cells from each cell line. Results were obtained from triplicate samples (mean $\pm \mathrm{SD})$.

in 3 groups (each 8 ) of nude mice and the tumourigenicity of each inoculated cell line was investigated by measuring the tumour sizes. Visible tumours appeared in all animals of the entire 3 groups only 3-5 days after the injection of cells. But the growth speed and hence the sizes of tumours produced by different cells were very different. All mice were sacrificed 20 days after the inoculation. A representative tumourbearing mouse from each of the 3 groups inoculated with different cells is shown in Fig. 5A and the tumour mass resected, respectively, from each corresponding animal is shown in the inserts. Average volumes of tumours produced, respectively by control, G-Id3-1 and G-Id3-7 cells in nude mice at different time-points during the inoculation period are shown in Fig. 5B. After the first week of inoculation the control cell line started to grow much faster than both transfected cell lines. On days 11, 14, 18 and 20, the average measured volumes of the tumours produced by control cells were all significantly larger than those produced by G-Id3-1 and G-Id3-7 at the corresponding time-points. No significant difference was found between the average volumes of tumours produced by G-Id3-1 and those produced by G-Id3-7 cells at any testing time-points. Measured at autopsy, the average volume of tumours produced by the control group was $1012 \pm 394 \mathrm{~mm}^{3}$. Whereas those produced by G-Id3-1 and G-Id3-7 cells were reduced by 2.1 - and 2.8-fold to $475 \pm 167$ and $356 \pm 178$, respectively. The average weight of tumours developed in each of the 3 groups of mice at autopsy is shown in Fig. 5C. While the average weight in the control group was $859 \pm 377 \mathrm{mg}$, the average weights in tumours produced by G-Id3-1 and G-Id3-7 cells were $436 \pm 167$ and $355 \pm 256 \mathrm{mg}$, respectively. Thus, in comparison to the control, the weights of tumours produced by G-Id3-1 and G-Id3-7 cells were significantly (Student t-test, $\mathrm{p}<0.026, \mathrm{p}<0.01$ ) reduced by 2.0- and 2.4-fold, respectively.

Effect of Id3 suppression on the sensitivity of cancer cells to apoptosis induction. The percentages of cells undergoing apoptosis were measured by detecting their outer membrane Annexin $\mathrm{V}$ through florescent flow cytometery and the results are shown in Fig. 6. When the cells were cultured in normal condition, there were $2.0,1.7$ and $2.3 \%$ of cells undergoing apoptosis in the control, G-Id3-1 and G-Id3-7 cells respectively (Fig. 6A); no significant difference. However, when apoptosis was induced by adding cytotoxin champtothecin $(40 \mu \mathrm{M})$ to the cultures, the percentage of apoptotic cells in the control transfectants was increased to $17.76 \%$, whilst those in G-Id3-1 and G-Id3-7 were increased to 41.51 and $45.75 \%$, respectively (Fig. 6B).

\section{Discussion}

SCLC has been described as a 'forgotten disease' (34) since the basic knowledge and treatment tactics for this cancer have faced a plateau for the past ten years. Many attempts to understand its molecular biology has been made, but little is found in terms of mechanisms involved in the rapid, disseminative nature of this devastating disease. Despite the encouraging early results regarding some new treatment strategies, no breakthrough has followed and new containment approaches have not increased survival time of treated patients. Therefore, the only way forward is to find new biomarkers and therapeutic targets based on novel molecular mechanisms involved in the initiation and progression.

We have previously found that all four Id proteins were generally up-regulated in SCLC tissue sections and cell lines and suggested that high levels of members of this protein family correlated with the malignant phenotype of SCLC. This indicated that Id proteins may play important roles in the pathogenesis of malignant progression and that they may be new potential targets for tumour suppression (1). This study has focused on Id3 protein, which showed the most pronounced over-expression amongst the Id-family proteins in SCLC, to test whether reduced expression of this protein can suppress the tumourigenicity of SCLC cells in vitro and in vivo. Amongst three candidate siRNA molecules, sequence-3 was identified by transient transfection as the most effective molecule to suppress Id3 expression in GLC-19 cells (Fig. 2A). By stable transfection of sequence-3, two cell lines (G-ID3-1 and G-Id3-7) with Id3 levels reduced to $\sim 30 \%$ of that in parental malignant SCLC cell line GLC-19 were established (Fig. 3A and B). The malignant characteristics 

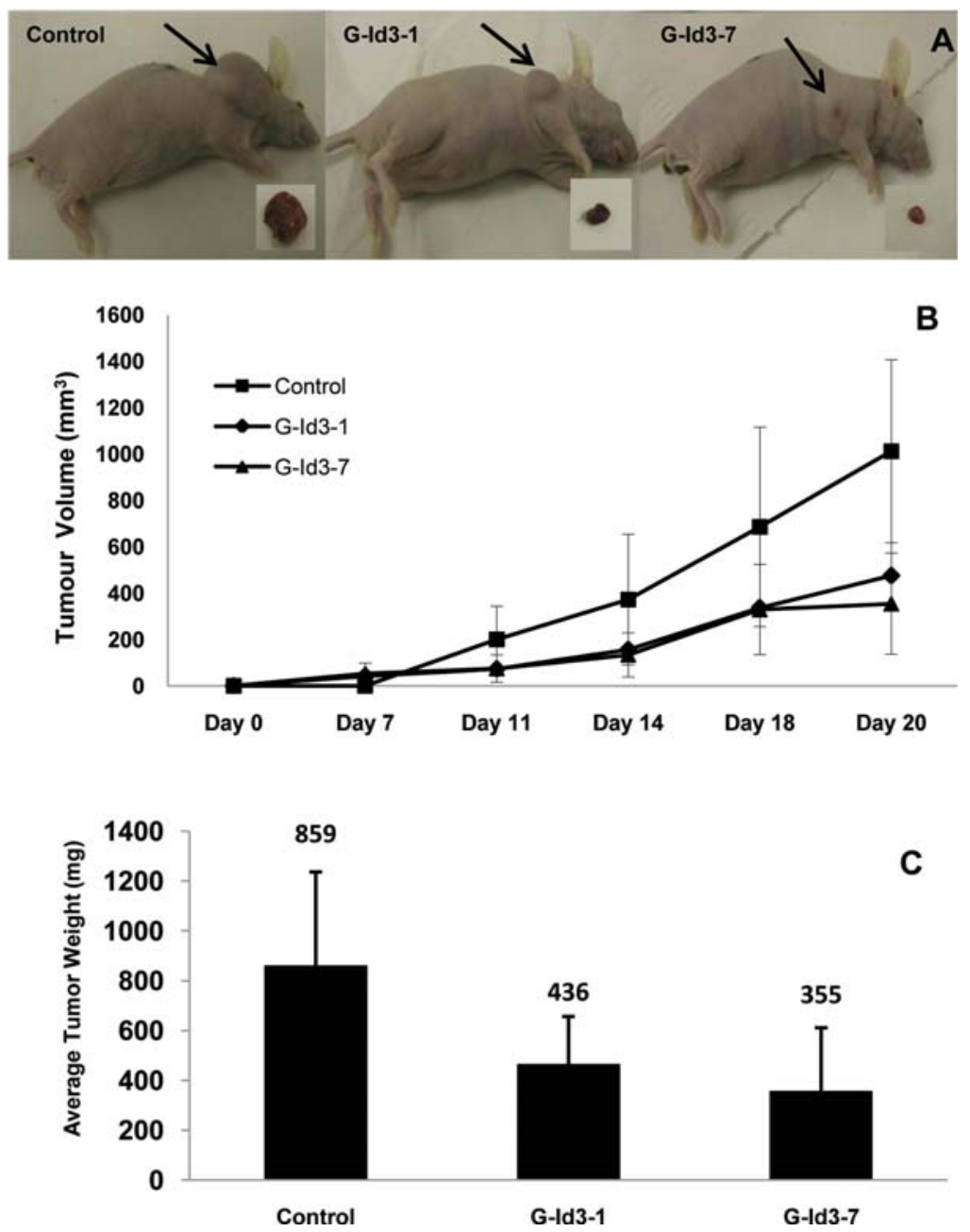

Figure 5. Tumourigenicity of control transfectants, G-Id3-1 and G-Id3-7 cells in nude mice. (A) A representative tumour-bearing mouse from each of the 3 groups of mice inoculated with different cells and tumour masses shown in the inserts were resected respectively from the animal shown in the same image. (B) Average volumes of tumours produced respectively by control, G-Id3-1 and G-Id3-7 cells in nude mice at different time-points during the inoculation period. For each group, 8 nude mice were used and tumours developed in all mice in 3 groups. Thus the average tumour volume in each group was measured from 8 mice (mean $\pm \mathrm{SD}$ ). (C) The average weight of tumours was obtained from each of the 3 groups of mice at autopsy (mean \pm SD).
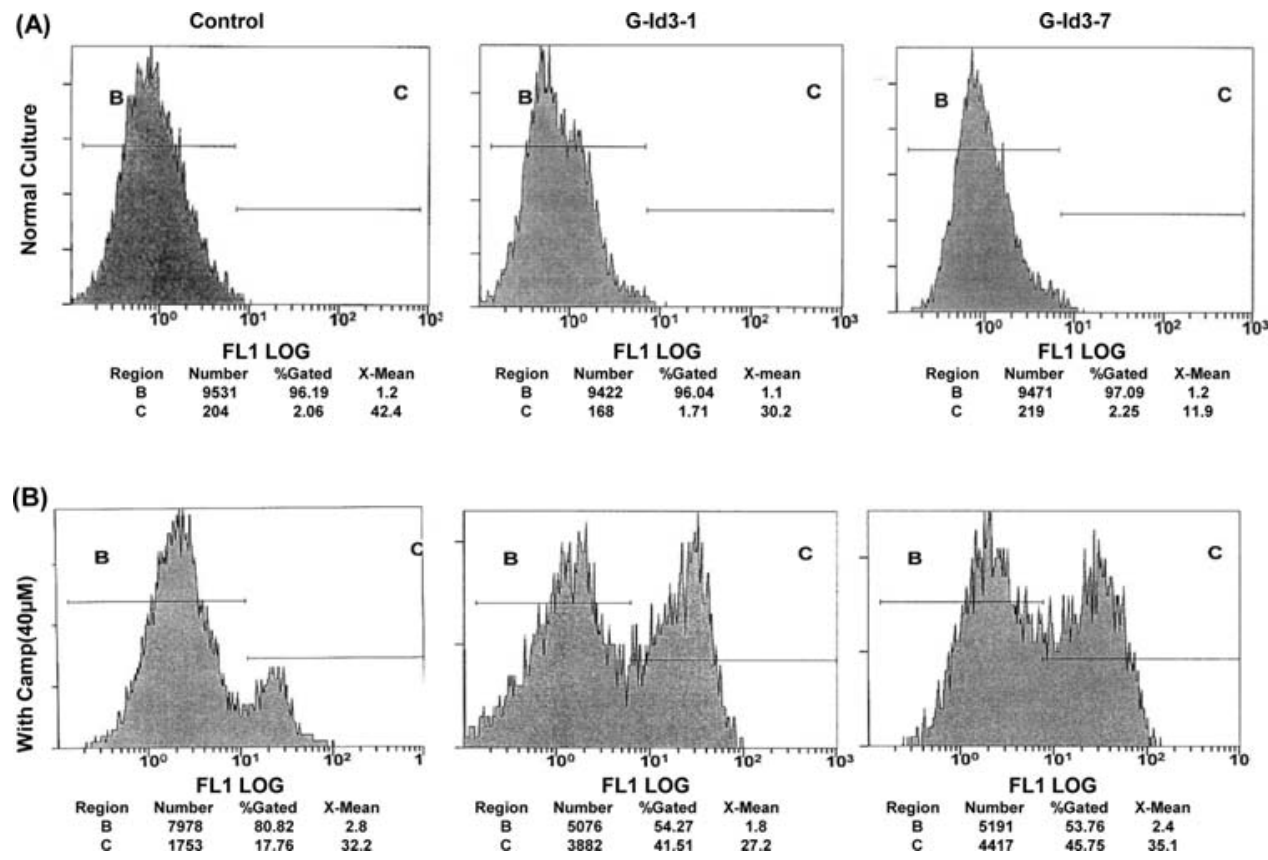

Figure 6. Percentages of cells undergoing apoptosis in control and si-Id3 transfectants growing in normal cultured condition without or with treatment by $40 \mu \mathrm{M}$ camptothecin. The peaks in (B) and (C) regions represent viable cells and cells undergoing apoptosis (or gated cells) respectively. 
of these cell lines were compared in vitro and in vivo to those of the control transfectant line generated by a scrambled RNA so as to assess the effect of the suppressed Id3 expression on the malignant progression of SCLC cells.

Bioassay results showed that suppression of $\mathrm{Id} 3$ expression in G-Id3-1 and G-Id3-7 cells produced significant reductions in proliferation rates by $>2$-fold (Fig. 4A). Soft agar assay results showed significant reductions in number of colonies produced by G-Id3-1 and G-Id3-7 cells comparing to that produced by the control. When inoculated into nude mice, the average volume of tumours produced by the control group was $1012 \pm 394 \mathrm{~mm}^{3}$. Whereas those produced by G-Id3-1 and G-Id3-7 cells were reduced by 2.1 - and 2.8 -fold to $475 \pm 167$ and $356 \pm 178$, respectively (Fig. 5B). While the average weight in the control group was $859 \pm 377 \mathrm{mg}$, those in tumours produced by G-Id3-1 and G-Id3-7 cells were 436 \pm 167 and $355 \pm 256 \mathrm{mg}$, respectively. Thus, in comparison to the control, the weights of tumours produced by G-Id3-1 and G-Id3-7 cells were significantly (Student's t-test, $\mathrm{p}<0.026, \mathrm{p}<0.01$ ) reduced by 2.0 - and 2.4-fold, respectively. The differences observed between the volumes and weights of the tumours were very similar, indicating that the two measurement methods are consistent. These results clearly showed that inhibition of Id3 expression in SCLC cells has significantly suppressed cell proliferation, anchorage-independent growth and tumourigenicity in nude mouse. Therefore, Id3 is a good candidate of a new therapeutic target for tumour suppression in SCLC.

Id3 has previously been shown to be involved in cell cycle regulation by one of the CDK inhibitor pathways such as p16 or p27. Disruption of Id3 and Id1 up-regulated p16 in mouse embryonic brain and resulted in impairment of neurogenesis (35). In most studies, Id3 was examined alongside Id 1 and their proliferative effects were linked together, except for a study using the xenopus model in which Id 3 was the only Id protein expressed during neural crest development. In this model, Id3 depletion caused absence of neural crest precursors and loss of neural crest derivatives due to cell cycle inhibition and cell death. Id3 depletion was accompanied by ectopic expression of cell cycle inhibitor p27 suggesting a negative regulatory role for Id 3 on p 27 transcription. Conversely, over-expression of Id 3 increased cell proliferation and resulted in expansion of the neural crest domain (36). Using a wounding model as an inducer of cell cycle entry for human dermal fibroblasts, when Id 3 was silenced, the expected down-regulation of p27 did not occurred, suggesting that transcription repression of p27 was regulated by Id3. It was also found that both G1 phase progression and G1/S transition were blocked by Id3 depletion (14).

In contrast to Id 2 that directly interacts with anti-proliferative RB protein, Id3 and Id1 exert their positive cell cycle regulatory effect indirectly by binding to and inhibiting ETS transcription factors or activating EGR-1 promoter. In this way, they inactivate CDK inhibitors such as p16, p21 or p27 which in turn, result in phosphorylation of $\mathrm{RB}$, liberation of Id2 and promote cell proliferation. MYC oncogene has been shown to utilize this effect in carcinogenesis by upregulating Id 3 protein. In this manner, Id 3 may suppress E2A factors involved in differentiation, growth suppression and apoptosis (37). Further proof to this notion was provided by an experiment on LNCaP, a weak prostate cancer cell line, where silencing Id3 resulted in enhancement of p16 and p21 levels, suggesting this was the Id3 knockdown that resulted proliferation suppression (38). In the present study, suppression of Id3 expression did not change the expression levels of either Id 1 or Id2 (Fig. 3C). Similar to that observed in prostate cancer, the tumourigenicity-suppression achieved in this study was also independent of levels of other Id proteins. However, since RB gene is mutated and inactivated in $98 \%$ of SCLC, it is unlikely that proliferation reduction in Id3-supressed cells is a result of RB re-activation. Further study is required to understand how the reduced Id 3 expression inhibited the malignant progression of SCLC cells.

Apart from the change in cell division rate through cell cycle regulation, alteration of apoptosis may also result in inhibition of cell proliferation and tumourigenicity. In this study, it was found that the percentages of apoptotic cells between si-Id3 cell lines and the control cell line were not significantly different (Fig. 6A) by Anexin-V labeling combined with flow cytometry. Thus inhibition of tumourigenicity by Id3-suppression may not be directly achieved by promoting apoptosis. When apoptosis was induced by a cytotoxin camptothecin, the percentages of cells undergoing apoptosis in culture G-Id3-1 and G-Id3-7 cells were increased by 2-3-fold comparing with that of the control cells (Fig. 6B). This result suggested that Id 3 may play a protective role in malignant cells when they are exposed to cytotoxins. Considering the fact that many anti-cancer drugs are apoptosis inducers, this finding could be important regarding treatment of this cancer and high level of Id3 may play a role in drug resistance in extensive disease. Further investigations are needed to determine the mechanisms and pathways through which Id3 affects proliferation and apoptosis in SCLC.

\section{Acknowledgements}

We thank Mrs. C. Beesley, Mr. T. Dickinson, and Mrs. P. Gerard for their technical assistance. This study was supported by a research project grant from North West Cancer Research Fund.

\section{References}

1. Kamalian L, Gosney JR, Forootan SS, et al: Increased expression of Id family proteins in small cell lung cancer and its prognostic significance. Clin Cancer Res 14: 2318-2325, 2008.

2. Perk J, Iavarone A and Benezra R: Id family of helix-loop-helix proteins in cancer. Nat Rev Cancer 5: 603-614, 2005.

3. Forrest $\mathrm{S}$ and McNamara C: Id family of transcription factors and vascular lesion formation. Arterioscler Thromb Vasc Biol 24: 2014-2020, 2004.

4. Benezra R, Davis RL, Lockshon D, Turner DL and Weintraub H: The protein Id: a negative regulator of helix-loop-helix DNA binding proteins. Cell 61: 49-59, 1990.

5. Moldes M, Boizard M, Liepvre XL, Feve B, Dugail I and Pairault J: Functional antagonism between inhibitor of DNA binding (Id) and adipocyte determination and differentiation factor $1 /$ sterol regulatory element-binding protein-1c (ADD1/ SREBP-1c) trans-factors for the regulation of fatty acid synthase promoter in adipocytes. Biochem J 344 : 873-880, 1999.

6. Pagliuca A, Gallo P, De Luca P and Lania L: Class A helix-loophelix proteins are positive regulators of several cyclin-dependent kinase inhibitors' promoter activity and negatively affect cell growth. Cancer Res 60: 1376-1382, 2000. 
7. Guo K, Wang J, Andres V, Smith RC and Walsh K: MyoDinduced expression of p21 inhibits cyclin-dependent kinase activity upon myocyte terminal differentiation. Mol Cell Biol 15: 3823-3829, 1995

8. French BA, Chow KL, Olson EN and Schwartz RJ: Heterodimers of myogenic helix-loop-helix regulatory factors and E12 bind a complex element governing myogenic induction of the avian cardiac alpha-actin promoter. Mol Cell Biol 11: 2439-2450, 1991.

9. Yates PR, Atherton GT, Deed RW, Norton JD and Sharrocks AD: Id helix-loop-helix proteins inhibit nucleoprotein complex formation by the TCF ETS-domain transcription factors. EMBO J 18: 968-976, 1999

10. Roberts EC, Deed RW, Inoue T, Norton JD and Sharrocks AD: Id helix-loop-helix proteins antagonize pax transcription factor activity by inhibiting DNA binding. Mol Cell Biol 21: 524-533, 2001

11. Lasorella A, Iavarone A and Israel MA: Id2 specifically alters regulation of the cell cycle by tumor suppressor proteins. Mol Cell Biol 16: 2570-2578, 1996.

12. Iavarone A, Garg P, Lasorella A, Hsu J and Israel MA: The helix-loop-helix protein Id-2 enhances cell proliferation and binds to the retinoblastoma protein. Genes Dev 8: 1270-1284, 1994.

13. Sherr CJ and Roberts JM: CDK inhibitors: positive and negative regulators of G1-phase progression. Genes Dev 13: 1501-1512, 1999.

14. Chassot AA, Turchi L, Virolle T, et al: Id3 is a novel regulator of p27(kip1) mRNA in early G1 phase and is required for cellcycle progression. Oncogene 26: 5772-5783, 2007.

15. Swarbrick A, Akerfeldt MC, Lee CS, et al: Regulation of cyclin expression and cell cycle progression in breast epithelial cells by the helix-loop-helix protein Id1. Oncogene 24: 381-389, 2005.

16. Light W, Vernon AE, Lasorella A, Iavarone A and La Bonne C: Xenopus Id3 is required downstream of Myc for the formation of multipotent neural crest progenitor cells. Development 132: 1831-1841, 2005.

17. Forootan SS, Wong YC, Dodson A, et al: Increased Id-1 expression is significantly associated with poor survival of patients with prostate cancer. Hum Pathol 38: 1321-1329, 2007.

18. Wilson JW, Deed RW, Inoue T, et al: Expression of Id helixloop-helix proteins in colorectal adenocarcinoma correlates with p53 expression and mitotic index. Cancer Res 61: 8803-8810, 2001.

19. Damdinsuren B, Nagano H, Kondo M, et al: Expression of Id proteins in human hepatocellular carcinoma: relevance to tumor dedifferentiation. Int J Oncol 26: 319-327, 2005.

20. Lin CQ, Singh J, Murata K, et al: A role for Id-1 in the aggressive phenotype and steroid hormone response of human breast cancer cells. Cancer Res 60: 1332-1340, 2000.

21. Schindl M, Oberhuber G, Obermair A, Schoppmann SF, Karner B and Birner P: Overexpression of Id-1 protein is a marker for unfavorable prognosis in early-stage cervical cancer. Cancer Res 61: 5703-5706, 2001.
22. Maruyama H, Kleeff J, Wildi S, et al: Id-1 and Id-2 are overexpressed in pancreatic cancer and in dysplastic lesions in chronic pancreatitis. Am J Pathol 155: 815-822, 1999.

23. Schindl M, Schoppmann SF, Strobel T, et al: Level of Id-1 protein expression correlates with poor differentiation, enhanced malignant potential, and more aggressive clinical behavior of epithelial ovarian tumors. Clin Cancer Res 9: 779-785, 2003.

24. Kebebew E, Treseler PA, Duh QY and Clark OH: The helixloop-helix protein, Id-1, is overexpressed and regulates growth in papillary thyroid cancer. Surgery 134: 235-241, 2003.

25. Langlands K, Down GA and Kealey T: Id proteins are dynamically expressed in normal epidermis and dysregulated in squamous cell carcinoma. Cancer Res 60: 5929-5933, 2000.

26. Ishiguro A, Spirin K, Shiohara M, et al: Expression of Id2 and Id3 mRNA in human lymphocytes. Leuk Res 19: 989-996, 1995.

27. Higashikawa K, Yoneda S, Tobiume K, et al: DeltaNp63alphadependent expression of Id-3 distinctively suppresses the invasiveness of human squamous cell carcinoma. Int J Cancer 124: 2837-2844, 2009

28. Shuno Y, Tsuno NH, Okaji Y, et al: Id1/Id3 knockdown inhibits metastatic potential of pancreatic cancer. J Surg Res 161: 76-82, 2010.

29. Adamson J, Morgan EA, Beesley C, et al: High-level expression of cutaneous fatty acid-binding protein in prostatic carcinomas and its effect on tumorigenicity. Oncogene 22: 2739-2749, 2003.

30. Freedman VH and Shin SI: Cellular tumorigenicity in nude mice: correlation with cell growth in semi-solid medium. Cell 3: 355-359, 1974.

31. Zwiebel JA, Davis MR, Kohn E, Salomon DS and Kidwell WR: Anchorage-independent growth-conferring factor production by rat mammary tumor cells. Cancer Res 42: 5117-5125, 1982.

32. Rygaard K and Spang-Thomsen M: Quantitation and gompertzian analysis of tumor growth. Breast Cancer Res Treat 46: 303-312, 1997.

33. Olsen MW, Ley CD, Junker N, Hansen AJ, Lund EL and Kristjansen PE: Angiopoietin-4 inhibits angiogenesis and reduces interstitial fluid pressure. Neoplasia 8: 364-372, 2006.

34. Rosti G, Carminati O, Monti M, Tamberi S and Marangolo M: Chemotherapy advances in small cell lung cancer. Ann Oncol 17 (Suppl. 5): 99-102, 2006

35. Lyden D, Young AZ, Zagzag D, et al: Id1 and Id3 are required for neurogenesis, angiogenesis and vascularization of tumour xenografts. Nature 401: 670-677, 1999.

36. Kee Y and Bronner-Fraser M: To proliferate or to die: role of Id3 in cell cycle progression and survival of neural crest progenitors. Genes Dev 19: 744-755, 2005.

37. Ruzinova MB and Benezra R: Id proteins in development, cell cycle and cancer. Trends Cell Biol 13: 410-418, 2003.

38. Asirvatham AJ, Carey JP and Chaudhary J: ID1-, ID2-, and ID3-regulated gene expression in E2A positive or negative prostate cancer cells. Prostate 67: 1411-1420, 2007. 